\title{
Analog VLSI Biophysical Neurons and Synapses With Programmable Membrane Channel Kinetics
}

\author{
Theodore Yu, Student Member, IEEE, and Gert Cauwenberghs, Senior Member, IEEE
}

\begin{abstract}
We present and characterize an analog VLSI network of 4 spiking neurons and 12 conductance-based synapses, implementing a silicon model of biophysical membrane dynamics and detailed channel kinetics in $\mathbf{3 8 4}$ digitally programmable parameters. Each neuron in the analog VLSI chip (NeuroDyn) implements generalized Hodgkin-Huxley neural dynamics in 3 channel variables, each with 16 parameters defining channel conductance, reversal potential, and voltage-dependence profile of the channel kinetics. Likewise, 12 synaptic channel variables implement a rate-based first-order kinetic model of neurotransmitter and receptor dynamics, accounting for NMDA and non-NMDA type chemical synapses. The biophysical origin of all 384 parameters in 24 channel variables supports direct interpretation of the results of adapting/tuning the parameters in terms of neurobiology. We present experimental results from the chip characterizing single neuron dynamics, single synapse dynamics, and multi-neuron network dynamics showing phase-locking behavior as a function of synaptic coupling strength. Uniform temporal scaling of the dynamics of membrane and gating variables is demonstrated by tuning a single current parameter, yielding variable speed output exceeding real time. The $0.5 \mu \mathrm{m}$ CMOS chip measures $3 \mathrm{~mm} \times$ $3 \mathrm{~mm}$, and consumes $1.29 \mathrm{~mW}$.
\end{abstract}

Index Terms-Neuromorphic engineering, reconfigurable neural and synaptic dynamics, silicon neurons, subthreshold metal-oxide semiconductor (MOS), translinear circuits.

\section{INTRODUCTION}

$\mathbf{N}$ EUROMORPHIC engineering [1] takes inspiration from neurobiology in the design of artificial neural systems in silicon integrated circuits (ICs), based on the function and structural organization of biological nervous systems. By emulating the form and architecture of biological systems, neuromorphic engineering seeks to emulate their function as well. Since the first silicon model of a biophysical neuron in 1990 [2], great advances have been made in the detail and scale of the modeling the neural function in silicon. Recently, the focus of the neuromorphic engineering effort in silicon modeling of the nervous system has shifted from the sensory periphery to central nervous

Manuscript received October 12, 2009; revised January 06, 2010. Current version published May 26, 2010. This work was supported by the National Institute of Aging, National Science Foundation, and Defense Advanced Research Projects Agency. This paper was recommended by Associate Editor P. Häfliger.

T. Yu is with the Department of Electrical and Computer Engineering, Jacobs School of Engineering, University of California, San Diego, CA 92039 USA. $\mathrm{He}$ is also with the Institute for Neural Computation, University of California, San Diego, CA 92039 USA (e-mail: teyu@ucsd.edu).

G. Cauwenberghs is with the Department of Bioengineering, Jacobs School of Engineering, University of California, San Diego, CA 92039 USA. He is also with the Institute for Neural Computation, University of California, San Diego, CA 92039 USA (e-mail: gert@ucsd.edu).

Color versions of one or more of the figures in this paper are available online at http://ieeexplore.ieee.org.

Digital Object Identifier 10.1109/TBCAS.2010.2048566

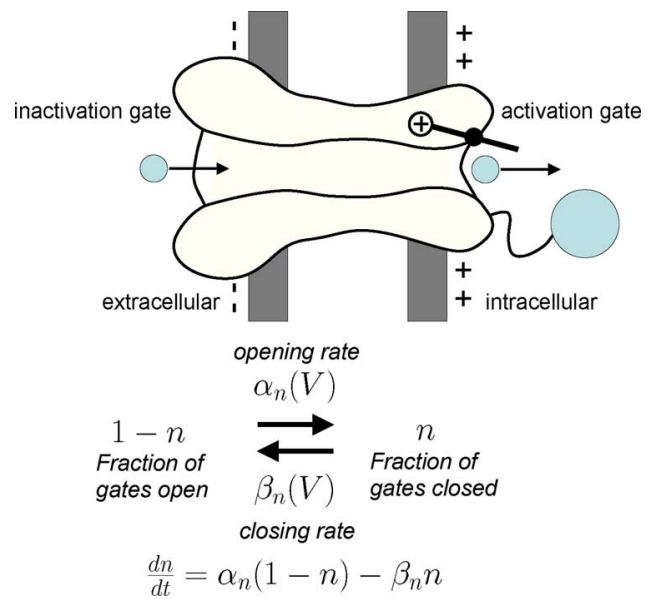

Fig. 1. An ion membrane channel is pictured [13] (top) with an accompanying mathematical expression (bottom) describing the channel kinetics in terms of the opening $\alpha$ and closing $\beta$ rates.

function addressing higher levels of integration and cognitive processing in the cortex and other brain regions [3]-[6], setting the stage for further advances toward closed-loop integration of biological and silicon neural systems [8]-[12].

Biophysical modeling and implementation of the neural function require a careful account of channel opening and closing kinetics and their role in ion transport through membranes that give rise to the rich neuronal and synaptic dynamics observed in neurobiology [13] (Fig. 1). Hodgkin and Huxley's seminal work in the investigation and formalization of neuron membrane dynamics have long been the standard of biophysical accuracy [14]. The difficulty of realizing the complex functional form of the Hodgkin-Huxley membrane currents and channel variables in analog circuits has motivated alternative realizations by simplifications in the model [15]-[18]. The prevailing approach in neuromorphic engineering design has been to abstract the neuron membrane action potential to discrete-time spike events in simplified models that capture the essence of integrateand-fire dynamics and synaptic coupling between large numbers of neurons in an address-event representation [19]-[25]. The advantage of these approaches is that they support event-based interchip communication, including direct input from neuromorphic audition [26] and vision [27] sensors, and may lead to highly efficient and densely integrated implementations in analog very-large scale integrated (VLSI) silicon [28], [29].

Here, we offer an alternative neuromorphic engineering approach that targets applications where biophysical detail in modeling neural and synaptic dynamics at the level of channel kinetics is critical. Examples of these applications include modeling of the effect of neuromodulators, neurotoxins, as well as neurodegenerative diseases on neural and synaptic 
function through parameter changes in the channel kinetics. For these and other applications in computational neuroscience, a direct correspondence between the parameters governing the biophysics of neural and synaptic function and those in the implemented computational model is greatly beneficial [30]-[33]. The approach we propose here is the first in analog VLSI to fully model the general voltage dependence of rate kinetics in the opening and closing of membrane ion channels. We illustrate this approach with the implementation and demonstration of NeuroDyn, an analog VLSI network of four neurons and 12 chemical synapses with a total of 384 digitally programmable parameters governing the channel conductance, reversal potential, and opening and closing kinetics voltage profile of 24 individually configurable channel variables.

Hodgkin and Huxley in their landmark paper [14] resorted to heuristics in curve-fitting the rate kinetics of channel variables observed through ingenious measurements on the squid giant axon. Any model replicating the precise functional form of this heuristic fit would, at best, produce an approximation to squid giant axons. To a large extent, the variety in dynamics between different neuron types in different organisms as well as the anomalies due to biomolecular agents and neurodegenerative processes acting on membrane channels arise from the resulting differences in channel properties. These channel properties are compactly characterized in our model by $16(7+$ $7+1+1)$ parameters for each channel variable specifying the voltage dependence of channel opening and closing rates (seven regression points each), besides values for the channel conductance and reversal potential. Fewer parameters would impair the flexibility in modeling neural and synaptic diversity in healthy and diseased nervous systems, although fewer parameters would be appropriate in special purpose implementations of specific model instances and their functional abstractions [34]-[38] where the application warrants efficiency rather than flexibility and biophysical explanatory power in parameter selections. Likewise, extensions to the models to incorporate further biophysical detail, such as short-term synaptic adaptation [39]-[42] and multicompartmental dynamics through linear [43] and nonlinear [44] dendritic coupling would incur larger numbers of parameters where the need for the extended models justifies the increase in implementation complexity.

While the implementation of parameterized channel kinetic rate equations in NeuroDyn provides the capacity to model a large variety of neuron and synapse behaviors, it requires tuning over a large number of parameters. Since each of these parameters has a direct physical correspondence in channel kinetics and membrane dynamics, values for these parameters can be obtained from physical considerations and measurements. Fine tuning of these parameters, to account for uncertainties in the modeling as well as imprecisions in the implemented model, would still be desirable. Extensive parameter fine-tuning was found to be unnecessary to address transistor mismatch. A simple calibration and parameter fitting procedure proved adequate to counteract mismatch and nonlinearities in setting parameters in the biophysical model to desired values. The correspondence between biophysical and circuit parameters is described in Section IV, with experimental alignment documented
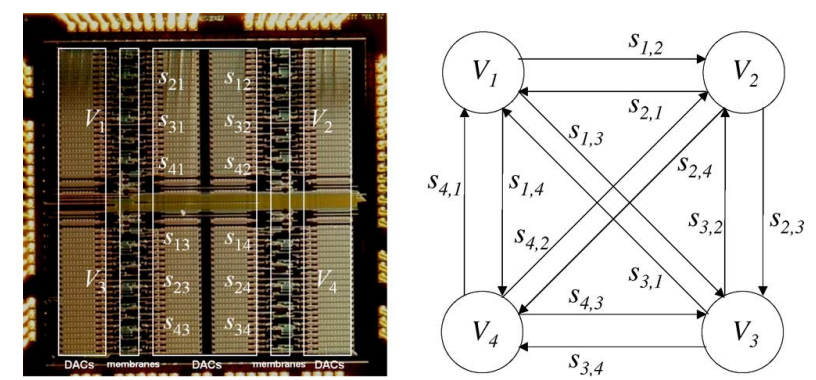

Fig. 2. NeuroDyn chip micrograph. (a, top left) and system diagram (b, top right). Four neurons are interconnected with 12 synapses, each with programmable channel kinetics, conductances, and reversal potentials (see Table I).

in Section $\mathrm{V}$ and the parameter alignment procedure detailed in Appendix B.

In the present implementation, we have aimed for functional flexibility and real-time control over parameters and internal dynamics, rather than efficiency and density of integration. The NeuroDyn system interfaces through the universal serial bus (USB) to Matlab software on a workstation to update the 384 parameters in rea time, and to continuously control and observe each of the four membrane potentials and 24 channel variables. To support this level of programmability, all parameters are stored locally on-chip in digital registers interfacing to a bank of 384 current multiplying digital-to-analog converters (DACs). The neuromorphic modeling approach presented here is extendable to other implementations where parameters may be shared across individual neurons and/or channels for greater efficiency, and combined with floating-gate nonvolatile analog storage [45]-[51] or dynamically refreshed volatile analog storage [52] of the parameters for greater density of integration. For example, central pattern generators require only a few neurons for implementation, yet they can characterize complex behavior [11].

We envisage NeuroDyn as an enabling tool for computational and systems neuroscience, since all internal dynamical variables and their parameters are grounded in the biophysics of membranes and ion channels. With its analog interface to the physical world, the NeuroDyn chip (Fig. 2) may also serve as an electronic training tool for budding neuroscientists and neurobiologists to practice patch clamp recording and other experimental techniques on "virtual" neurons. The NeuroDyn system contains various analog and digital exposed probes in the circuit board that allow for a real-time interface to the internal membrane potential and channel dynamics.

Furthermore, the low-power and efficient circuit implementation, combined with extensions for hardcoded parameter settings or high-density analog storage, support applications of the device as an implantable computational neural interface in vivo. These approaches, as described in [7], have great potential in the realization of intelligent neural prostheses when combined with embedded signal processing to process incoming neural spike data streams [8], [9] and activate prostheses [10], [12].

The analog VLSI design of the NeuroDyn system, and preliminary experimental results were presented in [53]. First results on coupled neural dynamics with inhibitory synapses were reported in [54]. Here, we provide details on the circuit implemen- 
TABLE I

NEURODYN DAC PARAMETERS

\begin{tabular}{rccc}
\hline Neurons $\mathrm{V}_{i}:$ & & & \\
$\alpha_{n_{i}}(V)$ & $\beta_{n_{i}}(V)$ & $\mathrm{g}_{N a_{i}}$ & $\mathrm{E}_{N a_{i}}$ \\
$\alpha_{m_{i}}(V)$ & $\beta_{m_{i}}(V)$ & $\mathrm{g}_{K_{i}}$ & $\mathrm{E}_{K_{i}}$ \\
$\alpha_{h_{i}}(V)$ & $\beta_{h_{i}}(V)$ & $\mathrm{g}_{L_{i}}$ & $\mathrm{E}_{L_{i}}$ \\
$4 \times 3 \times 7^{*}$ & $4 \times 3 \times 7^{*}$ & $4 \times 3$ & $4 \times 3$ \\
Synapses $\mathrm{s}_{i j}:$ & & & \\
$\alpha_{r_{i j}}\left(V_{p r e}\right)$ & $\beta_{r_{i j}}\left(V_{\text {post }}\right)$ & $\mathrm{g}_{s y n_{i j}}$ & $\mathrm{E}_{s y n_{i j}}$ \\
$12 \times 7^{*}$ & $12 \times 7^{*}$ & 12 & 12 \\
\hline
\end{tabular}

$*$ All rates $\alpha, \beta$ are functions of voltage as 7-point sigmoidal splines

(Section IV-A-1).

tation and complete experimental characterization of the neural and synaptic circuits, and present calibration and parameter-fitting procedures to align neural and synaptic characteristics from models or recorded data onto the digitally programmable analog hardware. We demonstrate the operation of the system by replicating opening and closing rates, gating variable kinetics, and action potentials of the Hodgkin-Huxley model, and study the dynamics of a network of two neurons coupled through reciprocal inhibitory synapses.

\section{NEURODYN ARCHITECTURE}

\section{A. System Overview}

The NeuroDyn board consists of four Hodgkin-Huxley (HH)based neurons fully connected through 12 conductance-based synapses as shown in Fig. 2(b). All parameters are individually addressable and individually programmable and are biophysically based governing the conductances, reversal potentials, and voltage dependence of the channel kinetics. There are a total of 384 programmable parameters governing the dynamics as shown in Table I. Each parameter is stored on-chip in a 10-b DAC.

\section{B. Chip Architecture}

The NeuroDyn chip is organized into four quadrants with each quadrant containing one neuron, and three synaptic inputs from the other neurons. Each neural and synaptic membrane channel current follows the same general form as illustrated in Fig. 3. Each channel current is a product of a conductance term modulated by a product of gating variables and the difference between the membrane voltage and reverse potential as in (2). The similar form for the neuron channel currents and synaptic current allows for a small number of circuits to model each component of the channel current.

\section{BIOPHYSICAL MODELS}

\section{A. Membrane Dynamics}

The HH membrane dynamics [14], including conductancebased synaptic input, are described by

$$
C_{m e m} \frac{d V_{i}}{d t}=-I_{N a_{i}}-I_{K_{i}}-I_{L_{i}}-\sum_{j} I_{s y n_{i j}}
$$

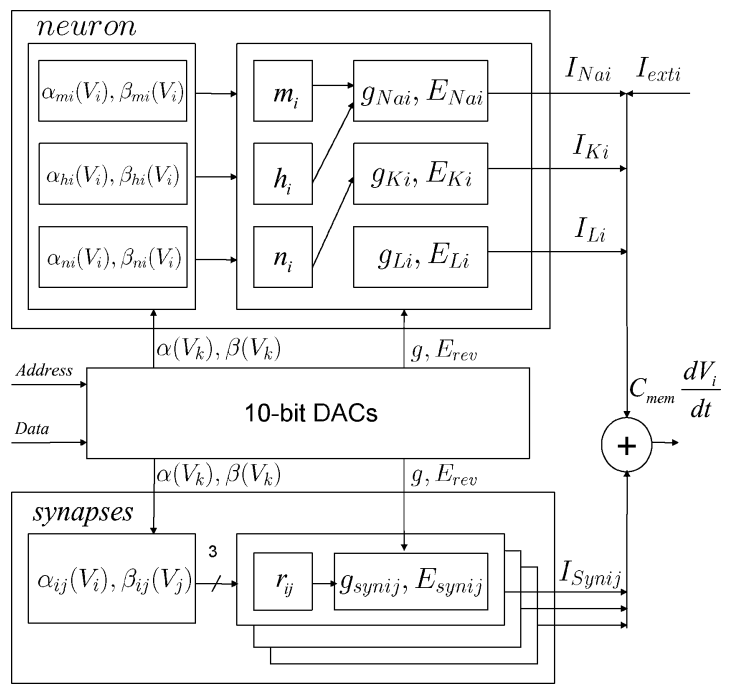

Fig. 3. System diagram for one of the four neurons in the NeuroDyn chip.

where $i, j=0 \ldots 3$, and

$$
\begin{aligned}
I_{N a_{i}} & =m_{i}{ }^{3} h_{i} g_{N a_{i}}\left(V_{i}-E_{N a_{i}}\right) \\
I_{K_{i}} & =n_{i}{ }^{4} g_{K_{i}}\left(V_{i}-E_{K_{i}}\right) \\
I_{L_{i}} & =g_{L_{i}}\left(V_{i}-E_{L_{i}}\right) \\
I_{s y n_{i j}} & =r_{i j} g_{s y n_{i j}}\left(V_{i}-E_{s y n_{i j}}\right) .
\end{aligned}
$$

All of the conductances in the model, including the synaptic conductances $g_{s y n_{i j}}$, are positive. Excitatory synapses are characterized by reversal potentials $E_{s y n_{i j}}$ above the rest potential, whereas for inhibitory synapses, the reversal potential $E_{s y n_{i j}}$ is below the rest potential.

\section{B. Channel Kinetics}

The neuron channel gating variables $n_{i}, m_{i}$, and $h_{i}$, as in the $\mathrm{HH}$ neuron formulation, are modeled by a rate-based first-order approximation to the kinetics governing the random opening and closing of membrane channels

$$
\begin{aligned}
\frac{d n_{i}}{d t} & =\alpha_{n_{i}}\left(1-n_{i}\right)-\beta_{n_{i}} n_{i} \\
\frac{d m_{i}}{d t} & =\alpha_{m_{i}}\left(1-m_{i}\right)-\beta_{m_{i}} m_{i} \\
\frac{d h_{i}}{d t} & =\alpha_{h_{i}}\left(1-h_{i}\right)-\beta_{h_{i}} h_{i}
\end{aligned}
$$

where the three channel variables $n_{i}, m_{i}$, and $h_{i}$ for each neuron $i$ denote the fractions of corresponding channel gates in the open state, and where the $\alpha$ and $\beta$ parameters are the corresponding voltage-dependent opening and closing rates.

Similarly, the synaptic channel currents are modeled by using first-order kinetics in the receptor variables $r_{i j}$, the fraction of receptors in the open state [55]

$$
\frac{d r_{i j}}{d t}=\alpha_{r_{i j}}\left(1-r_{i j}\right)-\beta_{r_{i j}} r_{i j}
$$

The opening rates $\alpha_{r_{i j}}$ are dependent on presynaptic voltage $V_{j}$, modeling the release of the neurotransmitter and its binding at the postsynaptic receptor, affecting the channel opening. In 


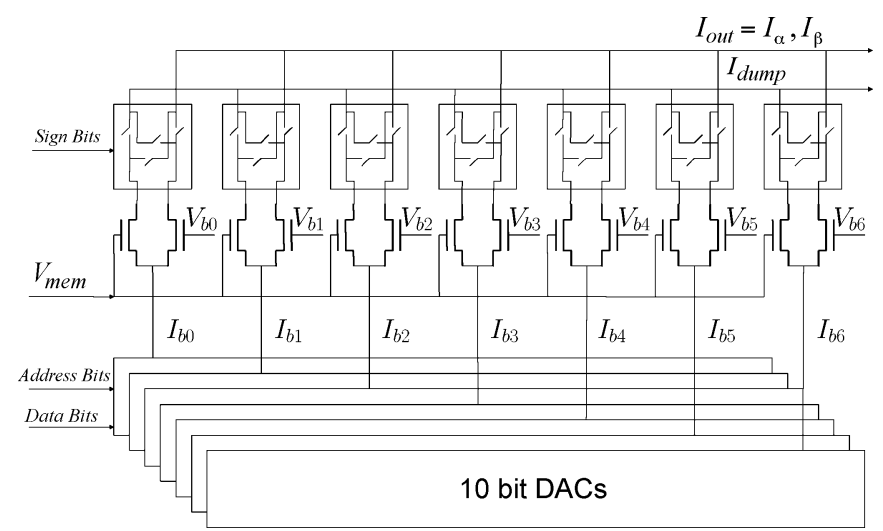

Fig. 4. Generalized channel rate variables $\alpha$ and $\beta$ implemented in the current domain with additive seven-point sigmoidal functions. Programmable parameters scaling the sigmoidal currents are stored in 10-b MOSFET-only R-2R DACs with additional bit governing polarity.

contrast, the closing rates $\beta_{r_{i j}}$ are generally dependent on postsynaptic voltage $V_{i}$. For non-NMDA synapses, this dependence is a constant, given the rate of unbinding and resulting decrease in the channel conductance following presynaptic deactivation. For NMDA synapses, this dependence models the effect of magnesium blocking of synaptic conductance triggered by postsynaptic potential.

The point of departure from the prevailing models in computational neuroscience is that the channel gate/receptor opening and closing rates are not specified and implemented as analytic functions, but are parameterized as regression functions, leaving significant flexibility in accommodating diversity in channel properties in the implemented model.

\section{NEUROMORPHIC IMPLEMENTATION AND CHARACTERIZATION}

\section{A. Voltage-Dependent Channel Kinetics}

1) Seven-Point Sigmoidal Spline Regression: Opening rates $\alpha$ and closing rates $\beta$ are modeled and regressed as 7-point additive spline sigmoidal functions implemented in the circuit illustrated in Fig. 4. Each sigmoid in the regression spline is implemented by a simple differential pair of metal-oxide semiconductor (MOS) transistors operating in subthreshold, where a bias current scales the sigmoid while a bias voltage determines the sigmoid offset [1]. These bias voltages are linearly spaced and are set through a voltage divider resistor string. A programmable 10-b metal-oxide semiconductor field-effect transistor (MOSFET)-only R-2R digital-to-analog converter (DAC) supplies the bias currents. An additional sign bit controls a switch circuit that determines the polarity of the output current slope, which selects either monotonically increasing or monotonically decreasing voltage dependence. The output currents from each differential pair are then additively combined to provide the composite function for the opening or closing rate. Each of the spline amplitudes and sign selection bits are individually programmable. By properly setting the current bias values and sign bit for each of the seven sigmoidal functions, the summation can accommodate a wide range of functions approximating typical rate functions $\alpha$ and $\beta$

$$
I_{\text {out }}(V)=\sum_{k=1}^{7} I_{b k} I_{\sigma, k}(V)=\sum_{k=1}^{7} \frac{I_{b k}}{1+e^{ \pm \kappa\left(V_{b k}-V\right) / V_{T}}}
$$

where the output current $I_{\text {out }}$ denotes either one of the $I_{\alpha}$ and $I_{\beta}$ rates, and where $V_{T}=k T / q$ is the thermal voltage.

To enforce a consistent temporal scale of the dynamics across membrane and gating variables, the currents implementing the opening and closing rates as well as the membrane conductances are globally scaled with a current $I_{\tau}$ that drives the multiplying DACs

$$
\begin{aligned}
I_{\alpha} & =\alpha I_{\tau} \\
I_{\beta} & =\beta I_{\tau} \\
I_{g} & =g I_{\tau}
\end{aligned}
$$

and, thus, uniformly controls the time base of all dynamic variables with a global temporal scale parameter $\tau=C V_{T} / I_{\tau}$.

2) Programmable Channel Kinetics: The gate opening and closing variables for one neuron were programmed to implement the $\mathrm{HH}$ model (Section III), with the target functions for the channel kinetics defined according to the $\mathrm{HH}$ opening and closing rate functions. The sigmoidal spline functions were measured from the chip to provide the basis functions at each spline location. Rectified linear least-squares optimization was then applied to determine the current bias parameters based on chip characteristics. Further parameter fitting details are provided in Appendix B. The 10-b programming for each of the seven spline amplitude levels in the regression functions results in the fit illustrated in Fig. 5. The closeness of fit is limited by the dynamic range of the 10-b DACs to simultaneously fit the steep slope of the $m_{\beta}$ gating variable and the gradual slopes of the $h_{\alpha}$ and $n_{\beta}$ parameters. Parameter fitting was achieved by applying rectified linear regression and iterative linear least-squares residue correction as described in Appendix B.

\section{B. Gated Conductances}

Gating variables $m_{i}, h_{i}, n_{i}$, and $r_{i j}$ are implemented as currents by the log-domain circuit shown in Fig. 6, which implements the kinetics (3)-(6) as

$$
C V_{T} \frac{d}{d t} \frac{I_{\text {out }}}{I_{\text {ref }}}=I_{\alpha}\left(1-\frac{I_{\text {out }}}{I_{\text {ref }}}\right)-I_{\beta} \frac{I_{\text {out }}}{I_{\text {ref }}}
$$

where $I_{\text {out }}$ represents the gating variable output current, and where $I_{\text {ref }}$ is a current reference that only affects the amplitude scale of the gating variables, but not the temporal scale of their dynamics.

The use of MOS transistors operating in the subthreshold region allows analog multiplication through the exponential relationship between the transistor input voltage and output current in translinear circuits [56]. The addition of the capacitor transforms the circuit from a translinear multiplier into a log-domain filter [59] that implements the desired first-order dynamics. The derivation is provided in Appendix A. 

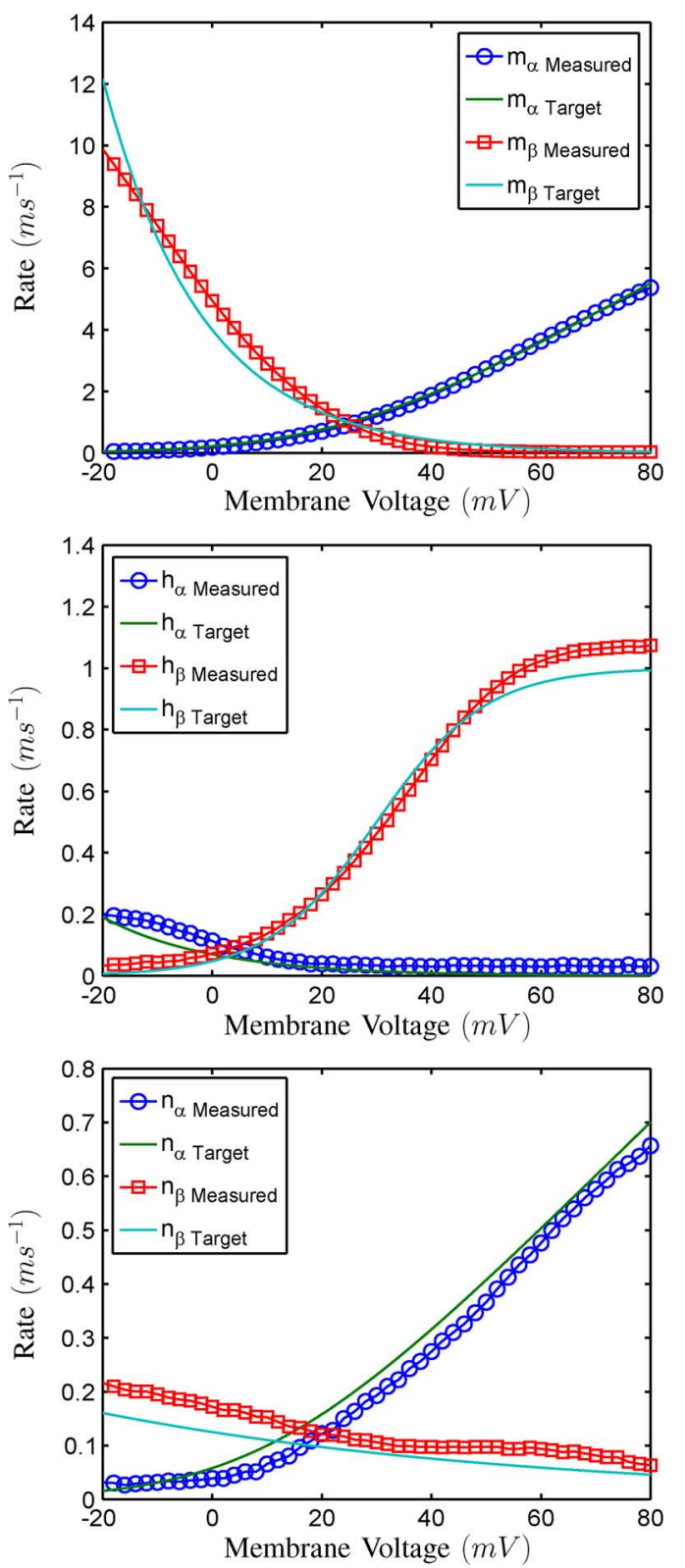

Fig. 5. Target and measured channel opening and closing rates $\alpha$ and $\beta$ for gating variables $n, m$, and $h$ of a single NeuroDyn neuron approximating the $\mathrm{HH}$ model, obtained by fitting the on-chip programmed parameters to the $\mathrm{HH}$ model.

The circuit is similar in implementation complexity to a previous implementation of rate kinetics [33] but avoids the backgate effect in the bulk CMOS process on the linearity in the first-order dynamics, and provides full programmability in the voltage profile of the dynamics. The circuit offers 14 parameters, specifying the detailed voltage dependence of the opening and closing rates offering flexibility in accurately modeling the channel kinetics.

1) Steady-State (in)Activation Functions: The steady-state (in)activation functions for one NeuroDyn programmed to replicate the HH model are shown in Fig. 7. These data were gathered by clamping the membrane voltage and slowly sweeping the membrane voltage while recording the values for the $m, h$, and $n$ gating variables. The results closely match the expected steady-state values according to the HH model [14]. Notice that

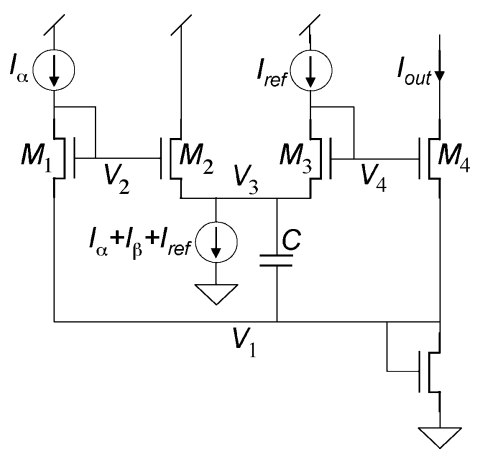

Fig. 6. Log-domain circuit implementing channel kinetics (3)-(6).
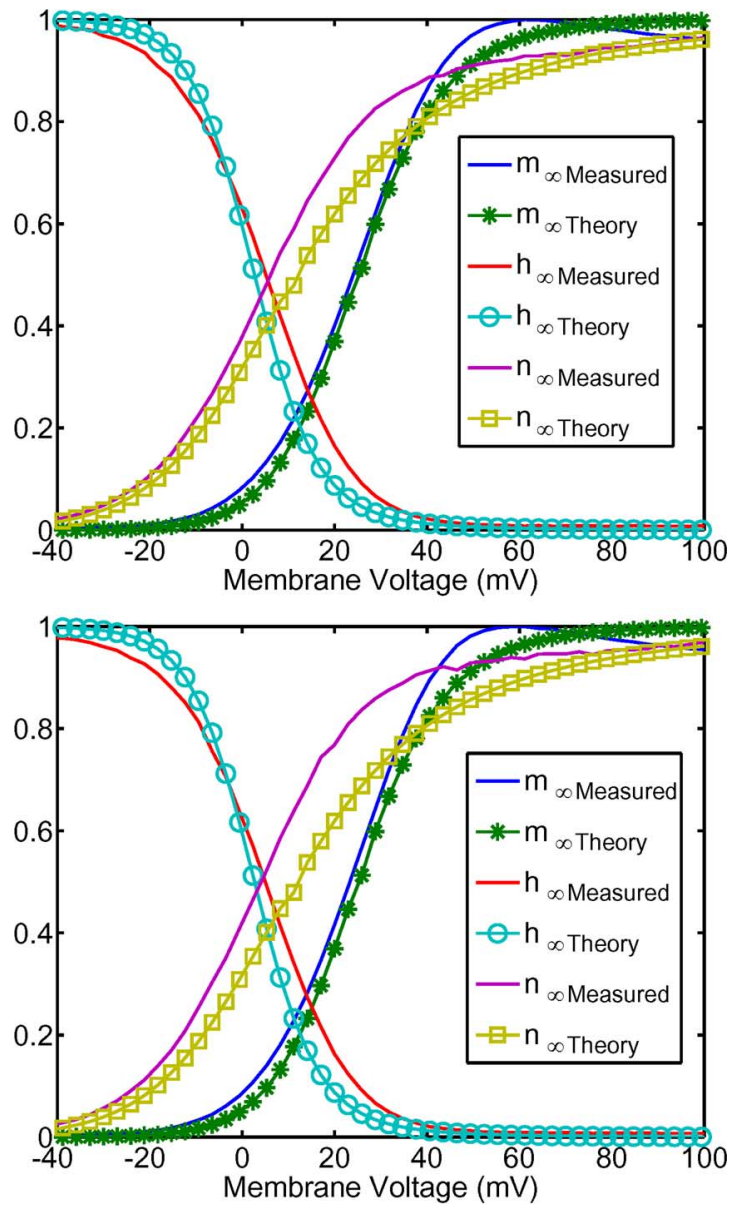

Fig. 7. Steady-state (in)activation functions measured on one neuron of $\mathrm{Neu}$ roDyn programmed to replicate the $\mathrm{HH}$ model (a) for fast setting of the neuron parameters and (b) for slow setting of the parameters, obtained without recalibration by increasing the global temporal scale parameter $I_{\tau} 2.5$-fold.

there is little variation between the fast and slow time-scale implementations obtained by varying the global temporal scale parameter $I_{\tau}$. This desirable time independence in the steady state (in)activation functions is clearly reflected in Fig. 7.

2) Voltage-Dependent Time Constants: The measured voltage-dependent time constants of the implemented $\mathrm{HH}$ model are shown in Fig. 8. The time constants were estimated by averaging the measured rise and fall times of changes in the gating variables under alternating small-amplitude voltage steps around the swept membrane voltage. The observed dynamics are consistent with the HH model [14] except for the 


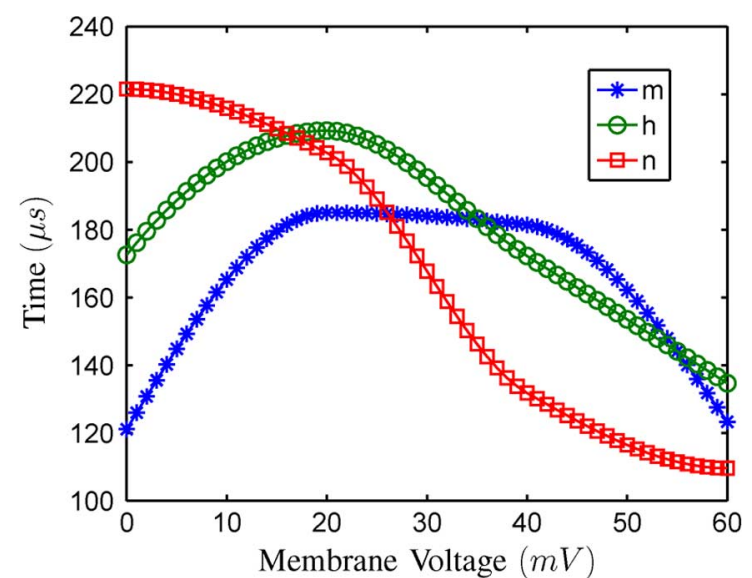

Fig. 8. Voltage-dependent time constants measured for one NeuroDyn neuron approximating the $\mathrm{HH}$ model.

larger observed time constants of the $m$ gating variable due to delays imposed by the on-chip output buffers.

\section{Translinear Multiplier}

A translinear multiplier shown in Fig. 9 implements gating of the membrane conductances with the gating variables. A translinear multiplier exploits the zero sum of voltages along a loop to implement a multiplication of current sources [57] and [56]

$$
I_{m^{3} h g_{N a}}=I_{g}\left(\frac{I_{m}}{I_{\text {ref }}}\right)^{3} \frac{I_{h}}{I_{\text {ref }}}
$$

where $I_{\text {ref }}$ is the same current reference controlling the amplitude of the gating variables (11) for dimensionless operation. Similar translinear circuits implement the other gated conductances of the form $x^{a}$ with $a+1$ stages, where $x=n$ and $a=4$ for the $\mathrm{K}+$ channel; and $x=r$ and $a=1$ for the conductance-based synapses.

1) Channel Conductance Dynamics: The channel conductance dynamics of an implemented $\mathrm{HH}$ model are shown in Fig. 10. The membrane voltage was clamped to the specified voltage levels and then released to measure the conductance dynamics for the $\mathrm{Na}+$ and $\mathrm{K}+$ channels. The results for the $\mathrm{Na}+$ channel show an increase in the magnitude and speed (as seen in the width of the curve) of the curve proportional to the magnitude of the depolarizing voltage step. The results for the $\mathrm{K}+$ channel also reflect an increase in magnitude and slope proportional to the magnitude of the depolarizing voltage step.

\section{Membrane Dynamics}

Each membrane conductance is implemented by a differential transconductance amplifier, linearized through shunting in the differential pairs for wide dynamic range in subthreshold MOS operation [58]. Unity gain connection of the amplifier yields a membrane current

$$
I_{N a}=\frac{\kappa}{V_{t h}} I_{m^{3} h g_{N a}}\left(V_{m}-E_{N a}\right) .
$$

For each of the membrane conductances, one amplifier is connected in parallel as shown in Fig. 11 . A capacitance $C_{\text {mem }}=C$ on the membrane node realizes the membrane dynamics (1).

\section{EXPERIMENTAL RESULTS}

\section{A. Neuron Spiking Dynamics}

We observed the dynamics of the membrane and gating variables for one neuron programmed to implement the $\mathrm{HH}$ model. We also demonstrated temporal control through the variation of the global temporal scale parameter set by current $I_{\tau}$. As shown in Fig. 12, the variation of $I_{\tau}$ scales the time axis of the waveforms by a factor greater than 2 . The amplitude scaling in the gating parameters reflects scaling proportional to $I_{\tau}$ consistent with (8)-(10). We implemented the HH model in one neuron and observed the dynamics of the membrane and gating variables as shown in Fig. 12. A small, constant $I_{\text {ext }}$ is applied to the neuron in order to provide dc input inducing spiking dynamics.

\section{B. Synapse Dynamics}

To observe the synapse dynamics, we took the spiking $\mathrm{HH}$ neuron from before and connected that as a presynaptic input to a synapse. The synapse parameters were configured to implement a $G A B A_{A}$ inhibitory synapse. The configuration is illustrated in Fig. 13(a). The conductance curves of the synapse are shown in Fig. 13(b). The synapse conductance curve was observed to rise quickly in time with the spiking neuron and slowly decay in accordance with the expected behavior.

\section{Neuron Network Dynamics}

We chose to demonstrate synaptic dynamics using a simple network of two neurons coupled with reciprocal inhibitory synapses as illustrated in Fig. 14. The neuron parameters were configured to implement the channel kinetic rate equations of the $\mathrm{HH}$ model. The synapse parameters were configured to implement $G A B A_{A}$ inhibitory synapses. The network was first initialized by disconnecting all of the synaptic connections by setting each synaptic conductance to zero. Then, separate external currents $I_{\text {ext1 }}$ and $I_{\text {ext2 }}$ were applied to the neurons $V_{1}$ and $V_{2}$, respectively, to induce spiking behavior. The values for $I_{\text {ext1 }}$ and $I_{\text {ext2 }}$ were chosen so that there was a small difference in the spiking frequency between the two neurons. Then, the synaptic conductances were increased until coupling was observed between the neurons, in the form of phase-locking. The resulting waveforms are shown in Fig. 15. Notice that especially in the oscilloscope capture from the coupled neurons, that there is observable timing jitter in the spiking neuron waveforms. This phase noise is primarily due to the noise intrinsic in the analog circuit implementation. Noise has also been observed in in vivo recordings of neuronal activity that can be attributed to thermal, stochastic, and other sources [60]. Thus, the noise from the circuit implementation may prove advantageous to provide a more biorealistic implementation.

\section{CONCLUSION}

We presented an analog VLSI network of biophysical neurons and synapses that implements general detailed models of 


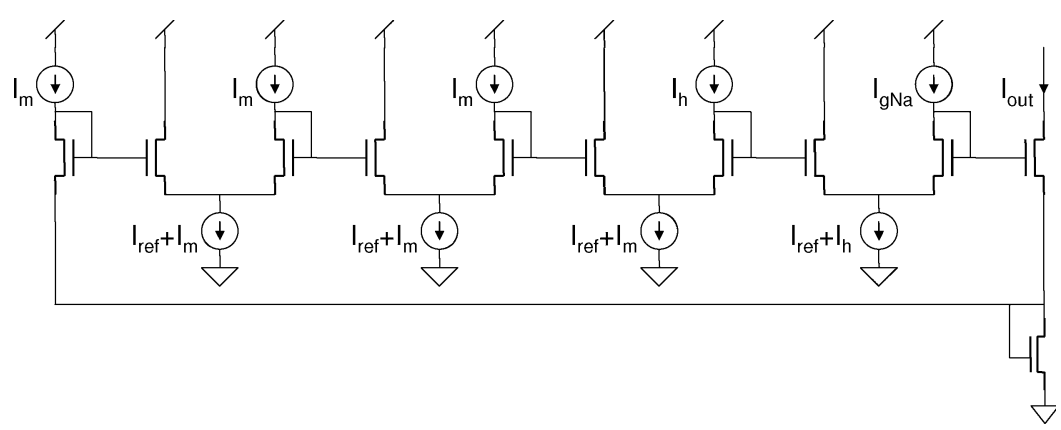

Fig. 9. Translinear circuit implementing gated conductances of the form $x^{3} y g$ such as $m^{3} h g_{N a}$ and $n^{4} g_{K}$. Synaptic gated conductances $r g_{s y n}$ are implemented by a two-stage version of the five-stage translinear circuit shown.
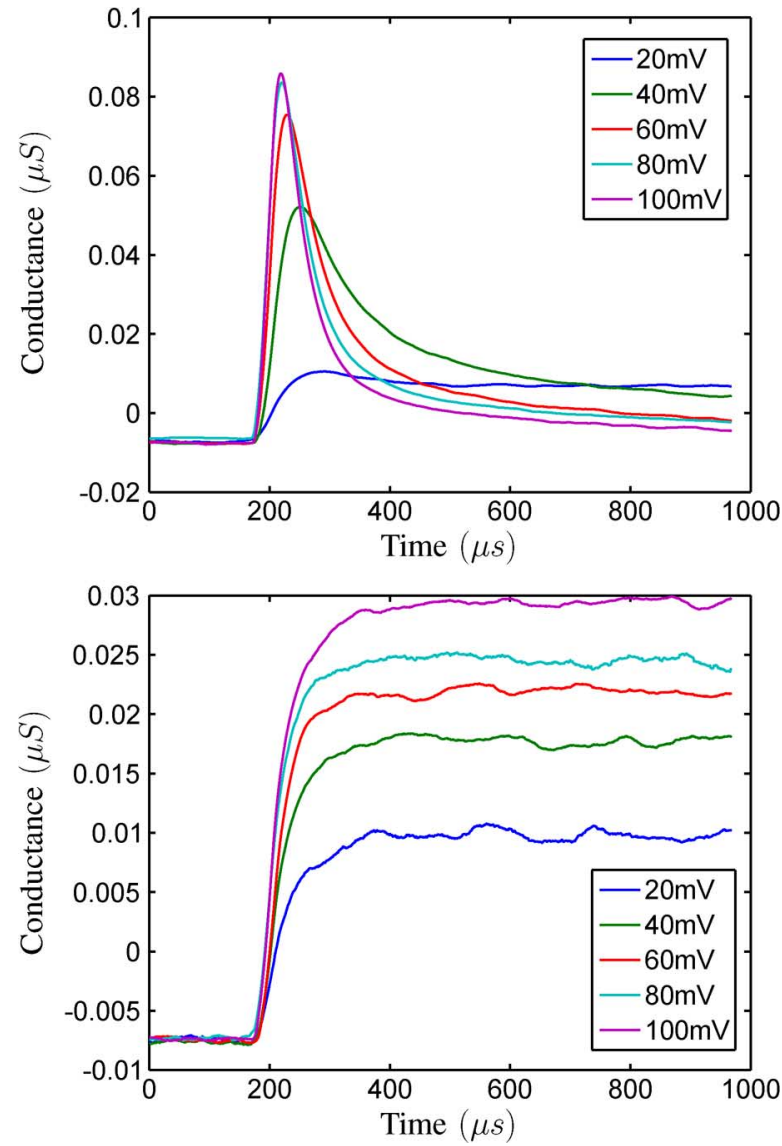

Fig. 10. Channel conductance dynamics measured from one NeuroDyn neuron approximating the $\mathrm{HH}$ model. (a) for the $\mathrm{Na}+$ channel and (b) for the $\mathrm{K}+$ channel. Channel conductance was measured for different depolarizing voltage steps away from the resting potential.

continuous-time membrane dynamics and channel kinetics in a fully digitally programmable and reconfigurable interface. Each neuron and synapse in the network offer individually programmable parameters setting reversal potentials, conductances, and voltage-dependent channel opening and closing rates. Least squares parameter fitting was shown to accurately reproduce biophysical neural data of channel opening and closing rates, gating variable dynamics, and action potentials. We further observed coupled neural spiking dynamics in a network with inhibitory synapses.

The implemented neural model extends on the $\mathrm{HH}$ formulation by allowing for arbitrary voltage profiles for channel

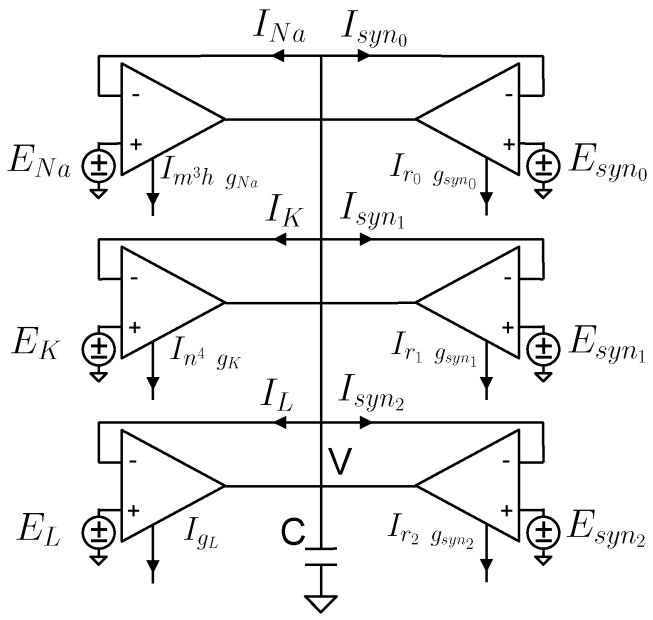

Fig. 11. Transconductance-C circuit implementing membrane dynamics for one neuron with synaptic input from the other three neurons.

opening and closing rates. The approach can be further extended by using similar principles to include adaptation mechanisms using calcium dynamics, and to implement resistively coupled multicompartment neurons. The work shown here represents a first step toward detailed silicon modeling of general neural and synaptic dynamics, combining digital and analog VLSI for maximum configurability and functionality.

\section{APPENDIX A}

\section{DERIVATION OF THE CIRCUIT IMPLEMENTING KINETICS OF CHANNEL GATING VARIABLES}

Here, we derive the dynamics of the circuit in Fig. 6 by implementing the kinetics in the channel gating variables by combining the $\alpha$ and $\beta$ rate currents. The log-domain circuit [59] uses the dynamic translinear principle, exploiting the exponential current-voltage dependence of MOS transistors operating in the subthreshold region [56]. Drain currents are modeled as $I_{d}=I_{0} W / L \exp \left(\left(\kappa V_{g}-V_{s}\right) / V_{T}\right)$ in gate voltage $V_{g}$ and source voltage $V_{s}$ relative to the bulk, where $V_{T}$ is the thermal voltage, $k T / q$ and $\kappa$ is the bulk back-gate effect factor [57]. The resulting translinear loop relation $I_{M_{1}} I_{M_{3}}=I_{M_{2}} I_{M_{4}}$ combined with Kirchhoff's current law leads to

$$
I_{\alpha} I_{r e f}=\left(I_{\alpha}+I_{\beta}+C \frac{d}{d t}\left(V_{3}-V_{1}\right)\right) I_{\text {out }} .
$$



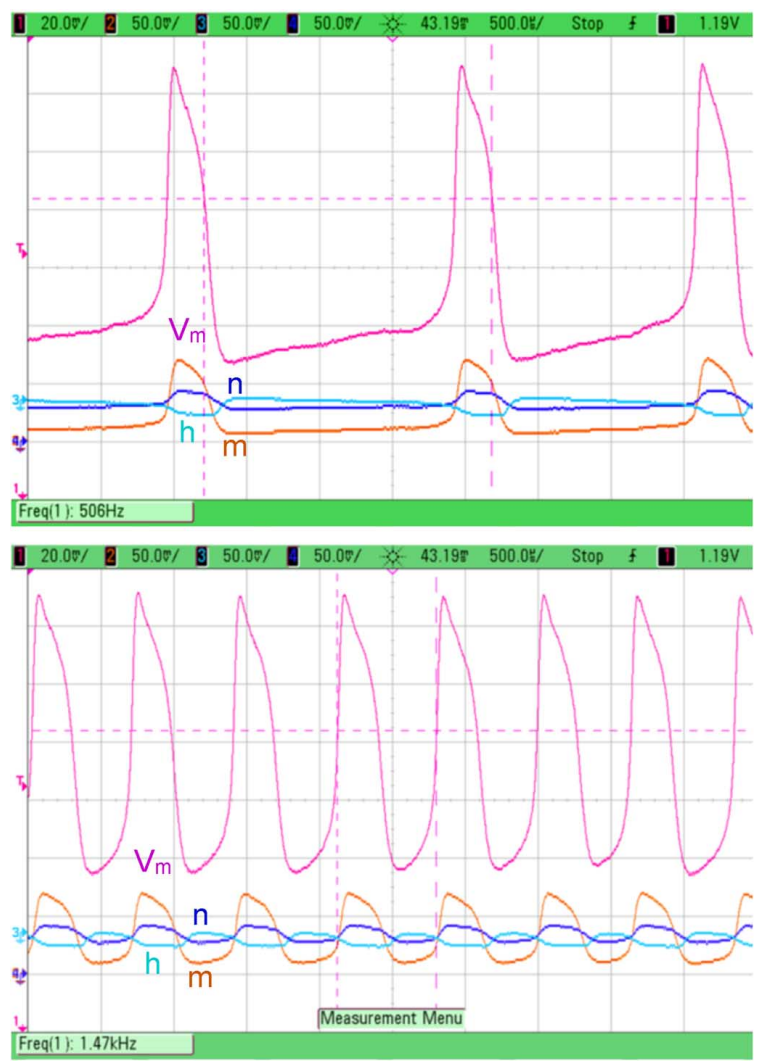

Fig. 12. Measured dynamics of membrane voltage $V_{m}$ and gating variables $n, m$, and $h$ for a single HH neuron. (a) and (b) show the effect of setting the global temporal scale parameter $I_{\tau}$, uniformly speeding or slowing the dynamics across all variables.

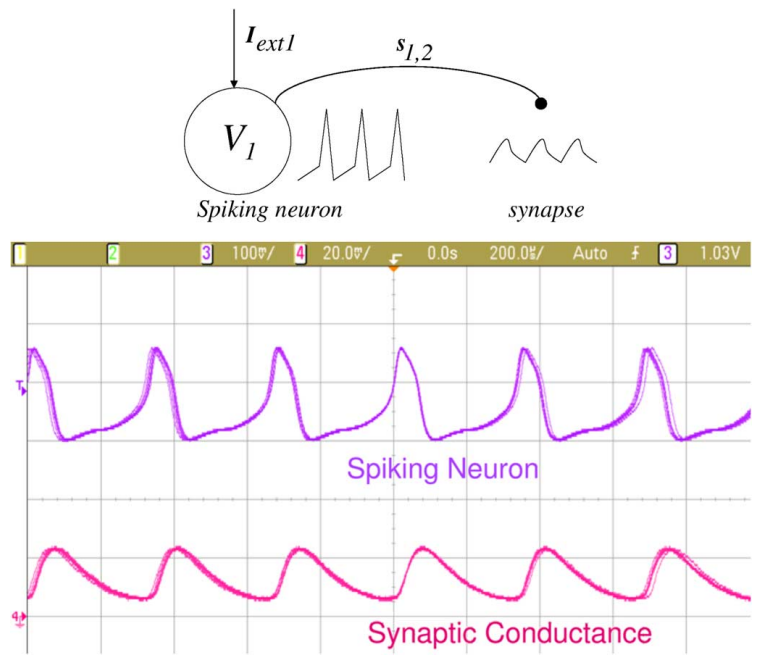

Fig. 13. (a) Synapse with presynaptic spiking neuron diagram (above). (b) Oscilloscope trace of the conductance curve of a synapse with a spiking presynaptic neuron input. Notice that the spiking neuron waveform (purple) and conductance curves for the synapse (pink) are in phase (below).

Since $I_{\text {out }} / I_{\text {ref }}=I_{M_{4}} / I_{M_{3}}=\exp \left(\kappa\left(V_{3}-V_{1}\right) / V_{T}\right)$, the voltage dynamics of $V_{3}-V_{1}$ in (14) are expressed in the current $\log$ domain as

$$
\frac{d}{d t} \frac{I_{\text {out }}}{I_{\text {ref }}}=\frac{\kappa}{V_{T}} \frac{d}{d t}\left(V_{3}-V_{1}\right) \frac{I_{\text {out }}}{I_{\text {ref }}}
$$

leading to (11).

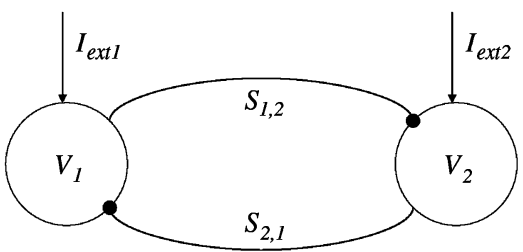

Fig. 14. Coupled neurons diagram. Two spiking neurons are connected with inhibitory synapses.

\section{APPENDIX B}

Calibration Procedure For $\alpha$ AND $\beta$ PARAmeter Fitting

\section{A. Rectified Linear Regression}

Let $I_{\text {meas }}\left(V, I_{b 1}, \ldots, I_{b 7}\right)$ be the measured $\alpha$ or $\beta$ function of $V$ obtained with current bias parameter settings $I_{b 1}, \ldots, I_{b 7}$. Then, for calibration, we measure the individual sigmoid contributions

$$
I_{\sigma, k}(V)=I_{\text {meas }}\left(V, \delta_{k 1}, \ldots, \delta_{k 7}\right)
$$

where $\delta_{k j}=1$ for $k=j$, and 0 otherwise. Hence, because of linearity in current summation, we may assume

$$
I_{\text {meas }}\left(V, I_{b 1}, \ldots, I_{b 7}\right) \approx \sum_{k=1}^{7} I_{b k} I_{\sigma, k}(V)
$$

To proceed, we perform a first linear fit of $I_{\text {meas }}\left(V, I_{b 1}, \ldots, I_{b 7}\right)$ to the target function $I_{\text {target }}(V)$ by using rectified linear leastsquares regression in the coefficients $I_{b k}$

$$
\min _{I_{b 1}, \ldots, I_{b 7} \geq 0}: \sum_{V}\left(\sum_{k=1}^{7} I_{b k} I_{\sigma, k}(V)-I_{\text {target }}(V)\right)^{2} .
$$

The rectification is necessary because of the positivity constraints on the bias current parameters.

\section{B. Iterative Linear Least-Squares Residue Correction}

Next, we correct for residual errors due to nonlinearities in the current multiplying DACs by implementing the sigmoid weighting (17). To do so, we linearize the system around the current operating point, by regressing the residue to locally differential sigmoid contributions

$$
\begin{aligned}
\Delta I_{\sigma, k}(V)=I_{\text {meas }}\left(V, I_{b 1}+\epsilon \delta_{k 1}, \ldots,\right. & \left.I_{b 7}+\epsilon \delta_{k 7}\right) \\
& -I_{\text {meas }}\left(V, I_{b 1}, \ldots, I_{b 7}\right)
\end{aligned}
$$

where $\epsilon$ is chosen sufficiently small for the linear analysis to be valid, but sufficiently large for reliable measurement. We proceed with another round of rectified linear least-squares regression in the parameters $I_{b k}+\Delta I_{b k}$ subject to the same positivity constraints, and iterate until the changes in parameter values $\Delta I_{b k}$ are small compared to the DAC precision.

\section{ACKNOWLEDGMENT}

The authors would like to thank S. Deiss and M. Chi for help with the experimental setup, and the MOSIS Educational Program for fabricating the chip. The authors also benefited 

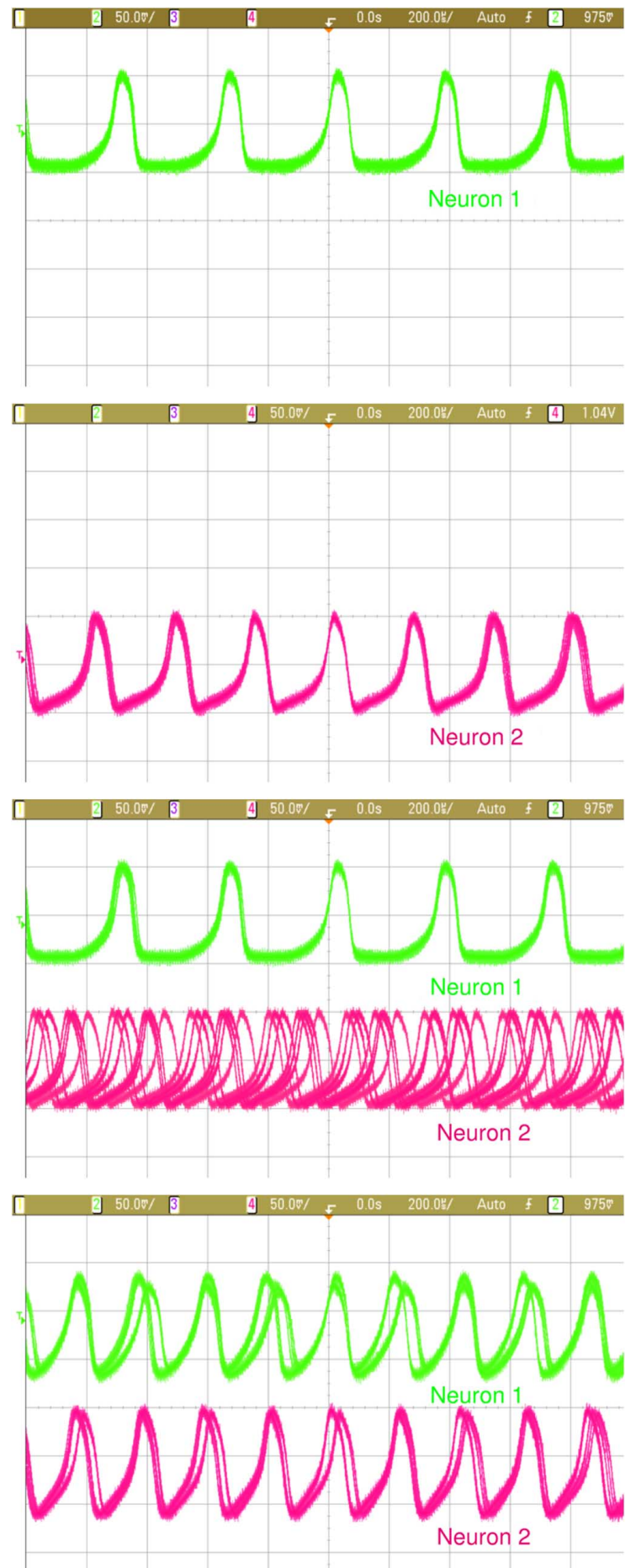

Fig. 15. Oscilloscope traces showing synaptic coupling in neural dynamics. (a) Through (c) individual uncoupled spiking neurons and (d) neurons coupled with inhibitory synapses spiking in synchrony.

from interactions with participants at the 2008 National Science Foundation Workshop on Neuromorphic Cognition Engineering in Telluride, CO.

\section{REFERENCES}

[1] C. A. Mead, Analog VLSI and Neural Systems. Reading, MA: Addison-Wesley, 1989.

[2] M. Mahowald and R. Douglas, "A silicon neuron," Nature, vol. 354, pp. 515-518, 1991.
[3] R. Serrano-Gotarredona, M. Oster, P. Lichtsteiner, A. Linares-Barranco, R. Paz-Vicente, F. Gomez-Rodriguez, K. Camunas-Mesa, R. Berner, M. Rivas-Perez, T. Delbruck, S. C. Liu, R. Douglas, P. Hafliger, G. Jimenez-Moreno, A. C. Ballcels, T. Serrano-Gotarredona, A. J. Acosta-Jimenez, and B. Linares-Barranco, "CAVIAR: A $45 \mathrm{k}$ neuron, $5 \mathrm{M}$ synapse, $12 \mathrm{G}$ connects/s AER hardware sensory-processing-learning-actuating system for high-speed visual object recognition and tracking," IEEE Trans. Neural Netw., vol. 20, no. 9, pp. 1417-1438, Sep. 2009.

[4] S. C. Liu and R. Douglas, "Temporal coding in a silicon network of integrate-and-fire neurons," IEEE Trans. Neural Netw., vol. 15, no. 5, pp. 1305-1314, Sep. 2004.

[5] R. J. Vogelstein, U. Mallik, E. Culurciello, G. Cauwenberghs, and R. Etienne-Cummings, "A multi-chip neuromorphic system for spike-based visual information processing," Neural Comput., vol. 19, no. 9, pp. 2281-2300, 2007.

[6] D. Sridharan, B. Percival, J. Arthur, and K. Boahen, "An in-silico neural model of dynamic routing through neuronal coherence," $A d v$. Neural Inf. Process. Syst., vol. 20, 2008.

[7] R. Sarpeshkar, Ultra Low Power Bioelectronics-Fundamentals, Biomedical Applications, and Bio-Inspired Systems. Cambridge, U.K.: Cambridge Univ. Press, 2010.

[8] M. C. Hsiao, C. H. Chan, V. Srinivasan, A. Ahuja, G. Erinjippurath, T. P. Zanos, G. Gholmieh, D. Song, J. D. Wills, J. LaCoss, S. Courellis, A R. Tanguay, J. J. Granacki, V. Z. Marmarelis, and T. W. Berger, "VLSI implementation of a nonlinear neuronal model: A 'Neural Prosthesis' to restore hippocampal trisynaptic dynamics," in Proc. IEEE Eng. Med. Bio Conf., 2006, pp. 4396-4399.

[9] R. Z. Shi and T. K. Horiuchi, "A neuromorphic VLSI model of bat interaural level difference processing for azimuthal echolocation," IEEE Trans. Circuits Syst. I, vol. 54, no. 1, pp. 74-88, Jan. 2007.

[10] T. A. Hudson, J. A. Bragg, P. Hasler, and S. P. DeWeerth, "An analog VLSI model of muscular contraction," IEEE Trans. Circuits Syst. II: Analog and Digital Signal Process., vol. 50, no. 7, pp. 329-342, Jul. 2003.

[11] R. J. Vogelstein, F. Tenore, L. Guevremont, R. Etienne-Cummings, and V. K. Mushahwar, "A silicon central pattern generator controls locomotion in vivo," IEEE Trans. Biomed. Circuits Syst., vol. 2, no. 3, pp. 212-222, Sep. 2008.

[12] V. Aggarwal, S. Acharya, F. Tenore, H. C. Shin, R. Etienne-Cummings, and N. V. Thakor, "Asynchronous decoding of dexterous finger movements using M1 neurons," IEEE Trans. Neural Syst. Rehab. Eng., vol. 16, no. 1, pp. 3-14, Feb. 2008.

[13] E. Kandel, J. Schwartz, and T. Jessell, Principles of Neural Science. New York: McGraw-Hill Medical, 2000.

[14] A. L. Hogdkin and A. F. Huxley, "A quantitative description of membrane current and its application to conduction and excitation in nerve," J. Physiol., vol. 117, pp. 500-544, 1952.

[15] B. Linares-Barranco, E. Sanchez-Sinencio, A. Rodriguez-Vazquez, and J. L. Huertas, "A CMOS implementation of FitzHugh-Nagumo neuron model," IEEE J. Solid-State Circuits, vol. 26, no. 7, pp. 956-965, Jul. 1991.

[16] D. Dupeyron, S. Le Masson, Y. Deval, G. Le Masson, and J. Dom, "A BiCMOS implementation of the Hodgkin--Huxley formalism," in Proc. 5th Int. Conf. Microelectronics for Neural Networks and Fuzzy Systems, 1996, pp. 311-316.

[17] J. Georgiou, E. M. Drakakis, C. Toumazou, and P. Premanoj, "An analogue micropower log-domain silicon circuit for the Hodgkin and Huxley nerve axon," in Proc. IEEE Int. Symp. Circuits Syst., 1999, vol. 2, pp. 286-289.

[18] M. F. Simoni, G. S. Cymbalyuk, M. E. Sorensen, R. L. Calabrese, and S. P. DeWeerth, "A multiconductance silicon neuron with biologically matched dynamics," IEEE Trans. Biomed. Eng., vol. 51, no. 2, pp. 342-354, Feb. 2004.

[19] K. A. Boahen, "Point-to-point connectivity between neuromorphic chips using address-events," IEEE Trans. Circuits Syst. II, vol. 47, no. 5, pp. 416-434, May 2000.

[20] G. Indiveri, E. Chicca, and R. Douglas, "A VLSI array of low-power spiking neurons and bistable synapses with spike-timing dependent plasticity," IEEE Trans. Neural Netw., vol. 17, no. 1, pp. 211-221, Jan. 2006.

[21] D. Badoni, M. Giulioni, V. Dante, and P. Del Giudice, "An aVLSI recurrent network of spiking neurons with reconfigurable and plastic synapses," in Proc. IEEE ISCAS , 2006, pp. 1227-1230.

[22] R. J. Vogelstein, U. Mallik, J. T. Vogelstein, and G. Cauwenberghs, "Dynamically reconfigurable silicon array of spiking neurons with conductance-based synapses," IEEE Trans. Neural Netw., vol. 18, no. 1, pp. 253-265, Jan. 2007. 
[23] R. Serrano-Gotarredona, T. Serrano-Gotarredona, A. Acosta-Jimenez, C. Serrano-Gotarredona, J. A. Perez-Carrasco, B. Linares-Barranco, A. Linares-Barranco, G. Jimenez-Moreno, and A. Civit-Ballcels, "On real-time AER 2-D convolutions hardware for neuromorphic spikebased cortical processing," IEEE Trans. Neural Netw., vol. 19, no. 7, pp. 1196-1219, Jul. 2008.

[24] S. Mitra, S. Fusi, and G. Indiveri, "Real-time classification of complex patterns using spike-based learning in neuromorphic VLSI," IEEE Trans. Biomed.Circuits Syst., vol. 3, no. 1, pp. 32-42, Feb. 2009.

[25] P. Livi and G. Indiveri, "A current-mode conductance-based silicon neuron for address-event neuromorphic systems," in Proc. IEEE ISCAS, 2009, pp. 2898-2901.

[26] V. Chan, S. C. Liu, and A. van Schaik, "AER EAR: A matched silicon cochlea pair with address event representation interface," IEEE Trans. Circuits Syst. I: Reg. Papers, vol. 54, no. 1, pp. 48-59, Jan. 2007.

[27] J. Costas-Santos, T. Serrano-Gotarredona, R. Serrano-Gotarredona, and B. Linares-Barranco, "A spatial contrast retina with on-chip calibration for neuromorphic spike-based AER vision systems," IEEE Trans. Circuits Syst. I: Reg. Papers, vol. 54, no. 7, pp. 1444-1458, Jul. 2007.

[28] H. Yang and R. Sarpeshkar, "A bio-inspired ultra-energy-efficient analog-to-digital converter for biomedical applications," IEEE Trans. Circuits Syst. I, vol. 53, no. 11, pp. 2349-2356, Nov. 2006.

[29] Y. Wong, P. Xu, and P. Abshire, "Ultra-low spike rate silicon neuron," in Proc. IEEE Biomedical Circuits Systems Conf., 2007, pp. 95-98.

[30] A. van Schaik, "Building blocks for electronic spiking neural networks," Neural Netw., vol. 14, no. 6-7, pp. 617-628, Jul.-Sep. 2001.

[31] E. Farquhar and P. Hasler, "A bio-physically inspired silicon neuron," IEEE Trans. Circuits Syst. 1: Reg. Papers, vol. 52, no. 3, pp. 477-488, Mar. 2005.

[32] S. Saighi, Y. Bornat, J. Tomas, and S. Renaud, "Neuromimetic ICs and system for parameters extraction in biological neuron models," in Proc. IEEE ISCAS, 2006, pp. 4207-4211.

[33] K. Hynna and K. Boahen, "Thermodynamically equivalent silicon models of voltage-dependent ion channels," Neural Comput., vol. 19, pp. 327-350, 2007.

[34] E. M. Izhikevich, "Simple model of spiking neurons," IEEE Trans. Neural Netw., vol. 14, no. 6, pp. 1569-1572, Nov. 2003.

[35] J. Wijekoon and P. Dudek, "Spiking and bursting firing patterns of a compact VLSI cortical neuron circuit," in Proc. Int. Joint Conf. Neural Networks, 2007, pp. 1332-1337.

[36] A. Basu, C. Petre, and P. Hasler, "Bifurcations in a silicon neuron," in Proc. IEEE ISCAS, pp. 428-431.

[37] A. Cassidy and A. G. Andreou, "Dynamical digital silicon neurons," in Proc. IEEE BioCAS, 2008, pp. 289-292.

[38] F. Folowosele, A. Harrison, A. Cassidy, A. Andreou, R. Etienne-Cummings, S. Mihalas, E. Niebur, and T. Hamilton, "A switched capacitor implementation of the generalized linear integrate-and-fire neuron," in Proc. IEEE ISCAS, 2009, pp. 2149-2152.

[39] E. Chicca, G. Indiveri, and R. Douglas, "An adaptive silicon synapse," in Proc. IEEE ISCAS, 2003, pp. I-81-I-84.

[40] J. Schemmel, A. Grubl, K. Meier, and E. Mueller, "Implementing synaptic plasticity in a VLSI spiking neural network model," in Proc. IEEE IJCNN, 2006, pp. 1-6, IEEE Press.

[41] C. Bartolozzi and G. Indiveri, "Synaptic dynamics in analog VLSI," Neural Comput., no. 19, pp. 2581-2603, 2007.

[42] Z. Yang, A. Murray, and J. Huo, "Deterministic coincidence detection and adaptation via delayed inputs," in ICANN (Part 2), 2008, pp. $453-461$.

[43] A. Basu, S. Ramakrishnan, C. Petre, S. Koziol, S. Brink, and P. Hasler, "Neural dynamics in reconfigurable silicon," IEEE Trans. Biomed. Circuits Syst., 2009, to be published.

[44] Y. Wang and S. C. Liu, "Input evoked nonlinearities in silicon dendritic circuits," in Proc. IEEE ISCAS, 2009, pp. 2894-2897.

[45] K. Kotani, T. Shibata, and T. Ohmi, "Neuron-MOS binary-logic circuits featuring dramatic reduction in transistor count and interconnections," in Proc. Int. Electron Devices Meeting Tech. Digest., Dec. 13-16, 1992, pp. 431-434.

[46] G. Cauwenberghs, C. F. Neugebauer, and A. Yariv, "Analysis and verification of an analog VLSI incremental outer-product learning system," IEEE Trans. Neural Netw., vol. 3, no. 3, pp. 488-497, May 1992.

[47] P. Hafliger and C. Rasche, "Floating gate analog memory for parameter and variable storage in a learning silicon neuron," in Proc. IEEE ISCAS, 1999, pp. 416-419.

[48] R. R. Harrison, J. A. Bragg, P. Hasler, B. A. Minch, and S. P. Deweerth, "A CMOS programmable analog memory-cell array using floating-gate circuits," IEEE Trans. Circuits Syst. II: Analog Digital Signal Process., vol. 48, no. 1, pp. 4-11, Jan. 2001.
[49] F. Tenore, R. J. Vogelstein, R. Etienne-Cummings, G. Cauwenberghs, and P. Hasler, "A floating-gate programmable array of silicon neurons for central pattern generating networks," in Proc. IEEE ISCAS, 2006, pp. 3157-3160.

[50] S. C. Liu and R. Mockel, "Temporally learning floating-gate VLSI synapses," in Proc. IEEE ISCAS, 2008, pp. 2154-2157.

[51] M. Pankaala, M. Laiho, and P. Hasler, "Compact floating-gate learning array with STDP," in Proc. Int. Joint Conf. Neural Networks, 2009, pp. 2409-2415.

[52] G. Cauwenberghs and A. Yariv, "Fault-tolerant dynamic multi-level storage in analog VLSI," IEEE Trans. Circuits Syst. II: Analog and Digital Signal Process., vol. 41, no. 12, pp. 827-829, Dec. 1994.

[53] T. Yu and G. Cauwenberghs, "Analog VLSI neuromorphic network with programmable membrane channel kinetics," in Proc. IEEE Int. Symp. Circuits and Systems, 2009, pp. 349-352.

[54] T. Yu and G. Cauwenberghs, "Biophysical synaptic dynamics in an analog VLSI network of Hodgkin-Huxley neurons," in Proc. IEEE Eng. Med. Biol. Conf., 2009, vol. 1, pp. 3335-3338.

[55] A. Destexhe, Z. F. Mainen, and T. J. Sejnowski, "Synthesis of models for excitable membranes, synaptic transmission and neuromodulation using a common kinetic formalism," J. Comp. Neurosci., vol. 1, pp. 195-230, 1994.

[56] A. Andreou and K. Boahen, "Translinear circuits in subthreshold MOS," Analog Integr. Circuits Signal Process., vol. 9, pp. 141-166, 1996.

[57] T. Serrano-Gotarredona, B. Linares-Barranco, and A. G. Andreou, "A general translinear principle for subthreshold MOS transistors," IEEE Trans. Circuits Syst. I: Fundam. Theory Appl., vol. 46, no. 5, pp. 607-616, May 1999.

[58] P. M. Furth and A. G. Andreou, "Linearised differential transconductors in subthreshold CMOS," Electron. Lett. Online, vol. 31, no. 7, pp. 545-546, 1995.

[59] T. Edwards and G. Cauwenberghs, "Synthesis of log-domain filters from first-order building blocks," J. Analog Integr. Circuits Signal Process., vol. 22, pp. 177-186, 2000.

[60] A. Manwani and C. Koch, "Detecting and estimating signals in noisy cable structures, I: Neuronal noise sources," Neural Comput., vol. 11, pp. 1797-1829, 1999.

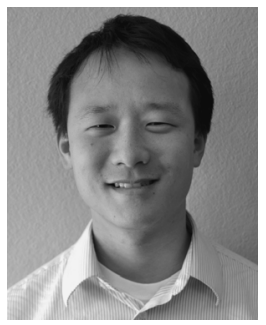

Theodore Yu (S'04) received the B.S. and M.S. degrees in electrical engineering from the California Institute of Technology, Pasadena, in 2004 and 2005, respectively, and is currently pursuing the Ph.D. degree in electrical and computer engineering at the University of California, San Diego.

His research interests include neuromorphic analog very-large scale integrated models of neural and synaptic circuits and circuit implementations of learning algorithms.

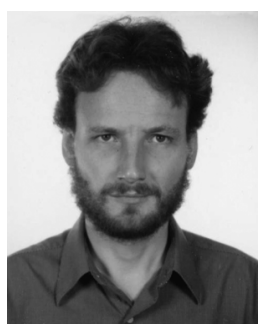

Gert Cauwenberghs (SM'89-M'94-SM'04) received the Ph.D. degree in electrical engineering from the California Institute of Technology, Pasadena.

Currently, he is Professor of Bioengineering at University of California, San Diego, where he co-directs the Institute for Neural Computation. Previously, he was Professor of Electrical and Computer Engineering at Johns Hopkins University, Baltimore, MD, and Visiting Professor of Brain and Cognitive Science at the Massachusetts Institute of Technology, Cambridge. His research interests cover neuromorphic engineering, computational and systems neuroscience, neuron-silicon and brain-machine interfaces, as well as learning and intelligent systems. He is Associate Editor for IEEE TRANSACTIONS ON BIOMEDICAL CiRCUITS AND SySTEMS, and IEEE TRANSACTIONS ON NEURAL SYSTEMS AND REHABILITATION ENGINEERING. He is a Senior Editor for the IEEE SENSORS JOURNAL.

Dr. Cauwenberghs received the National Science Foundation Career Award in 1997, the ONR Young Investigator Award in 1999, and Presidential Early Career Award for Scientists and Engineers in 2000. 